\title{
Uveal Melanoma and Secondary Angle-Closure Crisis: A Case Report and Literature Review
}

\author{
Tung Thanh Hoang ${ }^{a}$ b Tuan Anh Hoang ${ }^{c}$ Peter McCluskey ${ }^{b}$ \\ John Grigg ${ }^{b}$ \\ ${ }^{a}$ Ophthalmology Department, Hanoi Medical University Ophthalmology, Hanoi, Vietnam; \\ bSave Sight Institute, The University of Sydney Faculty of Medicine and Health, Sydney, \\ NSW, Australia; 'Ocular Pathology Department, Vietnam National Eye Hospital, Hanoi, \\ Vietnam
}

Keywords

Uveal melanoma $\cdot$ Secondary angle closure $\cdot$ Serous retinal detachment

\begin{abstract}
A 66-years-old Vietnamese healthy female patient presented with prolonged severe right ocular pain and complete vision loss in that eye. Anterior segment assessment including gonioscopy identified angle-closure configuration. A suspected ciliary body melanoma was seen through the pupil. Posterior segment examination revealed a large tumor mass and $360^{\circ}$ retinal detachment (kissing configuration). An ultrasound examination was consistent with a uveal tumor. The painful, blind right eye with a tumor mass was enucleated. Histopathology confirmed a type A uveal spindle cell melanoma associated with total serous retinal detachment without evidence of tumor necrosis, epithelioid cells, scleral, or optic nerve infiltration. There was no evidence of metastasis after 1-year of follow-up. It is critically important to differentiate primary and secondary angle closure, especially in cases with life-threatening ocular malignancy as uveal melanoma.
\end{abstract}

\section{Introduction}

Identifying the etiology of angle closure is critical for instituting the appropriate management. Ocular hypertension as a presenting sign of intraocular tumors has been described in several studies, but angle closure due to choroidal melanoma appears far less common than other mechanisms such as invasion or seeding of the angle, iris neovascularization, and intraocular hemorrhage, or associated with other ocular tumor types [1-4]. In this study, we report a uveal melanoma manifesting as angle-closure crisis and total serous retinal detachment. 


\section{Case Report}

A 66-years-old Vietnamese female patient acutely presented with severe pain and no light perception in the right eye. The symptoms began 2 months ago, with progressive loss of vision, haloes, and ipsilateral headache. Her medical and family history was noncontributory.

Examination of the right eye revealed vision of no perception of light, diffuse conjunctival injection, corneal edema, shallow anterior chamber, fixed dilated right pupil, no RAPD, elevated intraocular pressure of $30 \mathrm{~mm} \mathrm{Hg}$, age-related nuclear cataract, and $360^{\circ}$ retinal detachment (kissing configuration) (Fig. 1). Left eye examination was normal. B-scan ultrasonography revealed a right superior intraocular mass arising from the choroid and ciliary body and with a poorly defined scleral margin (Fig. 2).

The clinical diagnosis was right angle-closure crisis secondary to uveal tumor and complete retinal detachment. As the right eye was blind and painful, an enucleation was performed. There was an intraocular, $1.2 \mathrm{~cm}$, black mass arising from the choroid on macroscopic examination (Fig. 3a, b). Histopathologic examination revealed a type A spindle cell melanoma (Fig. 4a) with an occluded iridocorneal angle (Fig. 4b), total serous retinal detachment. There was no tumor necrosis, epithelioid cells, scleral (Fig. 4c), or optic nerve (Fig. 4d) infiltration. There was no evidence of metastasis after 1 year of follow-up.

\section{Discussion}

Primary angle-closure crisis is common in patients of Asian [5] and Vietnamese ethnicities [6]. This patient presented with typical features of this entity and might have been misdiagnosed without careful posterior segment examination.

Fig. 1. Kissing choroid.

Fig. 2. Uveal tumor with a poorly defined scleral margin.
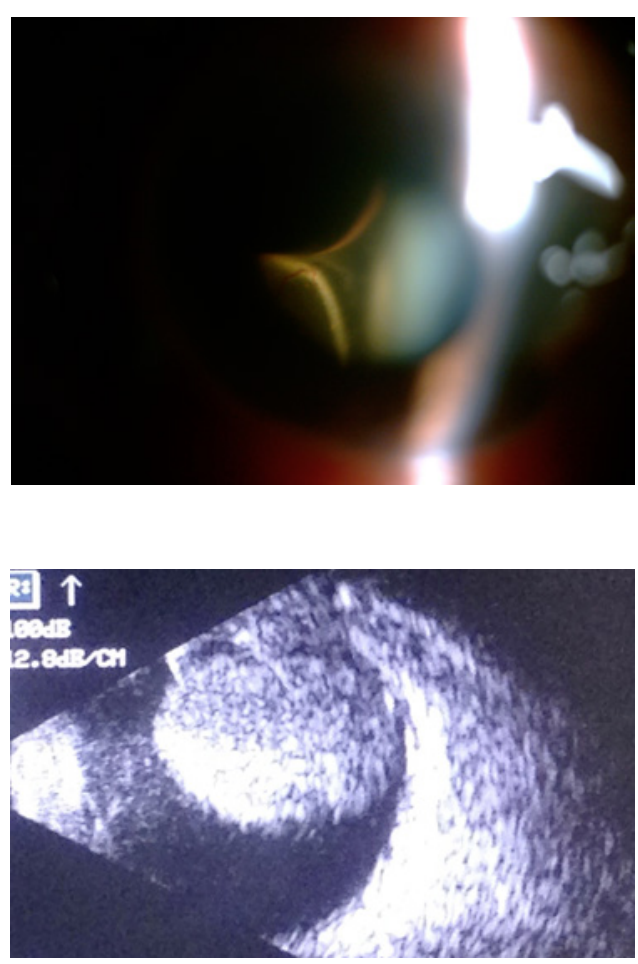

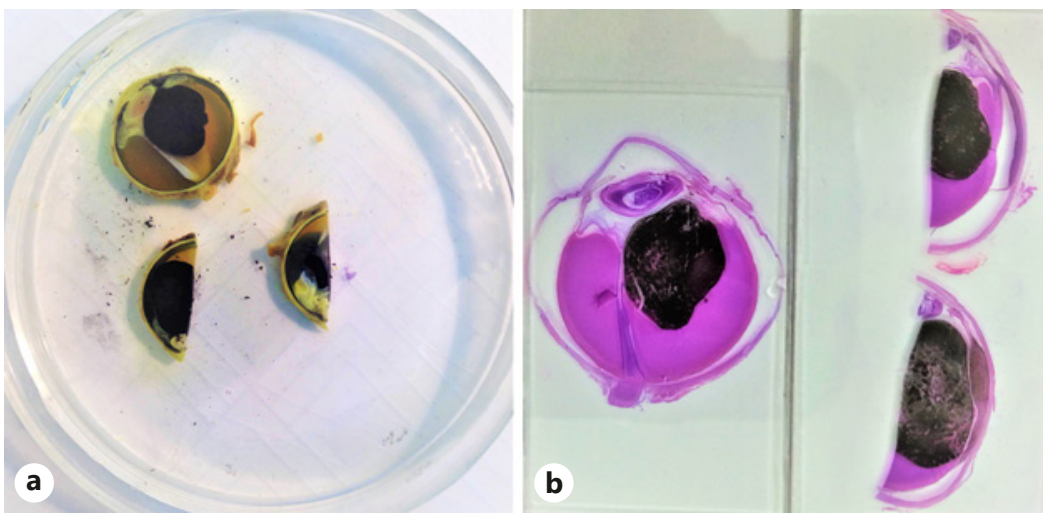

Fig. 3. a Sagital view of the enucleated eye (black mass attaching to sclera wall). b Macroscopic view of the tumor ( $\mathrm{H}$ and $\mathrm{E}$ stain).
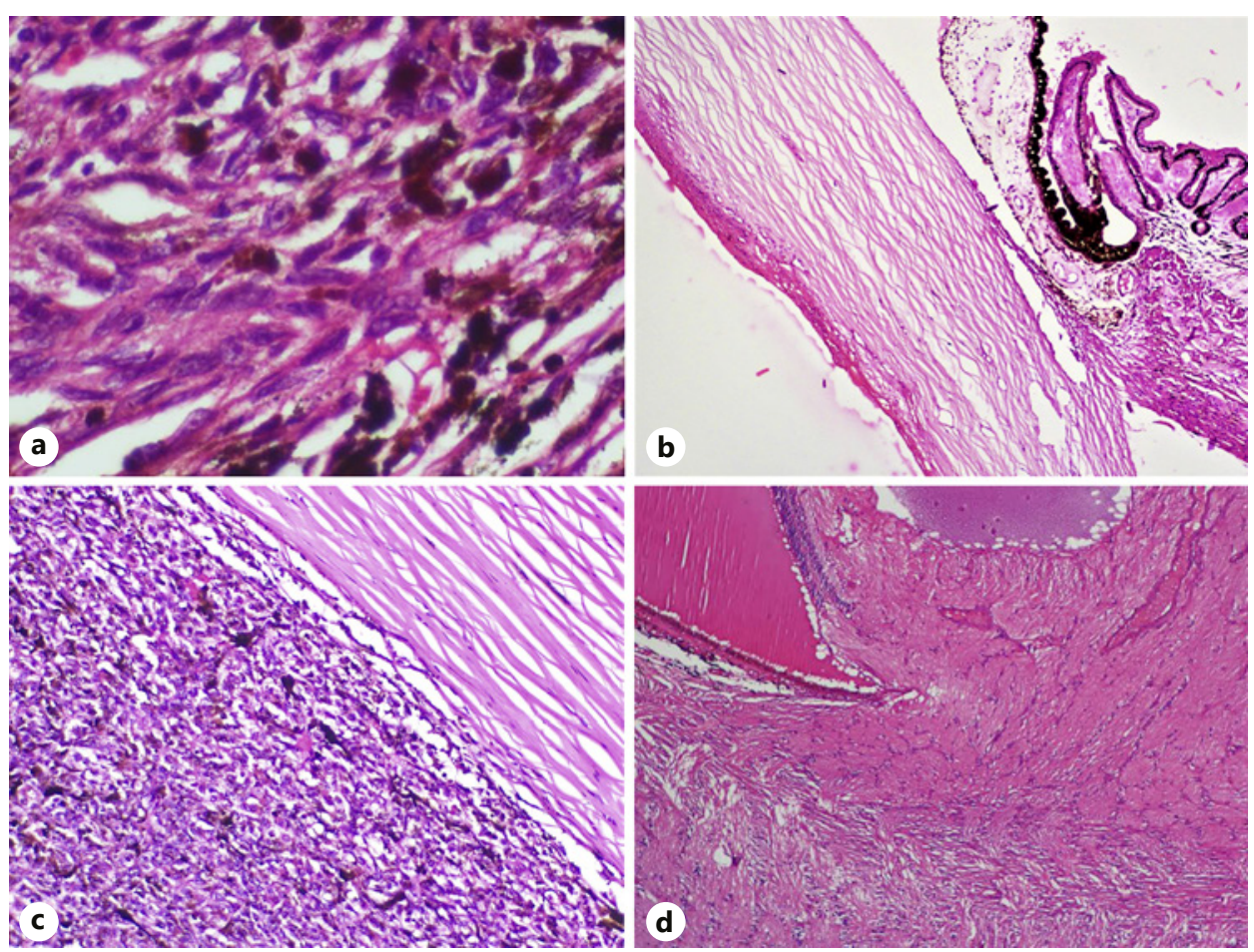

Fig. 4. a Type A spindle cell melanoma. b Closed iridocorneal angle. c No scleral invasion. $\mathbf{d}$ No optic nerve invasion.

Secondary angle-closure crisis can happen with (lens-induced disorders) or without pupillary block (anterior pulling or posterior pushing distortions of the lens-iris interface). Vascular membrane, hemorrhage, inflammation process (exudate, derbis, and keratic precipitates) or epithelial ingrowth might lead to the formation of peripheral anterior synechie, causing the closure of iridocornea angle. Contrarily, intraocular tumors, malignant glaucoma, or uveal effusion may put a pressure on the iris posteriorly, leading to the contact with the trabecular meshwork. When considering the angle-closure mechanism in this patient, it could be the mass effect of the tumor inducing the anterior displacement of the lens-iris diaphragm, subsequently closing the angle. Therefore, a thorough posterior segment examination was important to confirm the diagnosis. 
Schwartz et al. [7] describe a choroidal melanoma manifesting as an acute angle-closure event that was diagnosed 1 week after laser iridotomy and medical intervention. Singer et al. [8] reported another melanoma case with acute unilateral angle-closure glaucoma that underwent a filtration procedure. The melanoma was not diagnosed for $>2$ weeks after the first visit. Consequent to the surgery, the tumor seeded into the orbit and subsequently metastasized to the liver with the patient dying 2 years after diagnosis [8].

Palamar et al. [9] and Iturralde et al. [10] described cases with secondary angle closure to choroidal melanoma associated with choroidal effusion due to tumor necrosis. In contrast, histopathological examination of our patient revealed serous retinal detachment which is common in uveal melanoma [11] without evidence of tumor necrosis. Mearza et al. [12] reported a patient with a suspicious choroidal nevus that after 8 years presented with a choroidal neovascular membrane that induced total retinal detachment and angle closure following a massive subretinal hemorrhage. Subsequent pathology identified a low-grade spindle cell melanoma [12].

Uveal tumors may be primary ocular malignant neoplasms or secondary metastases. Uveal melanoma is the commonest uveal tract malignancy and its prognosis is variable with spindle A cell melanoma having the best prognosis with a 15-year mortality rate of $19 \%[13]$.

\section{Conclusions}

Identifying the etiology for angle closure is essential. In this case, the detailed assessment identified a choroidal melanoma as the cause of angle closure, which is critical for managing patients with a life-threatening ocular malignancy.

\section{Acknowledgements}

All the authors express the deepest gratitude to Dr. Cuong Hoang Nguyen, Vietnam National Eye Hospital, for having successfully performed the enucleation procedure.

\section{Statement of Ethics}

The reportstrictly follows the tenets of World Medical Association Declaration of Helsinki. The consent form has been provided and signed by the patient.

\section{Conflict of Interest Statement}

The authors have no conflicts of interest to declare.

\section{Funding Sources}

The authors certify that they have no affiliations with, or involvement in, any organization or entity with any financial or nonfinancial interest in the subject matter discussed.

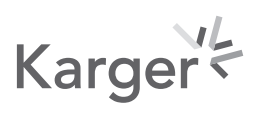


Hoang et al.: Uveal Melanoma and Secondary Angle-Closure Crisis

\section{Author Contributions}

Tung Thanh Hoang was in charge of examining the patient before and after the surgery, reviewing the medical literature, and writing the manuscript. Tuan Anh Hoang assessed the histopathological samples. Peter McCluskey and John Grigg reviewed and edited the manuscript. All the authors approved the final version of the manuscript for publication.

\section{References}

1 Shields CL, Shields JA, Shields MB, Augsburger JJ. Prevalence and mechanisms of secondary intraocular pressure elevation in eyes with intraocular tumors. Ophthalmology. 1987 Jul;94(7):839-46.

2 Othman IS, Assem M, Zaki IM. Secondary glaucoma as initial manifestation of uveal melanoma. Saudi J Ophthalmol. 2013;27(3):203-8.

3 Camp DA, Yadav P, Dalvin LA, Shields CL. Glaucoma secondary to intraocular tumors: mechanisms and management. Curr Opin Ophthalmol. 2019 Mar;30(2):71-81.

4 Vempuluru VS, Jakati S, Krishnamurthy R, Senthil S, Kaliki S. Glaucoma as the presenting sign of intraocular tumors: beware of the masquerading sign. Int Ophthalmol. 2020 Mar 20;40(7):1789-95.

5 Kapetanakis VV, Chan MP, Foster PJ, Cook DG, Owen CG, Rudnicka AR. Global variations and time trends in the prevalence of primary open angle glaucoma (POAG): a systematic review and meta-analysis. Br J Ophthalmol. 2016 Jan;100(1):86-93.

6 Peng PH, Manivanh R, Nguyen N, Weinreb RN, Lin SC. Glaucoma and clinical characteristics in Vietnamese Americans. Curr Eye Res. 2011 Aug;36(8):733-8.

7 Schwartz GP, Schwartz LW. Acute angle closure glaucoma secondary to a choroidal melanoma. CLAO J. 2002 Apr;28(2):77-9.

8 Singer PR, Krupin T, Smith ME, Becker B. Recurrent orbital and metastatic melanoma in a patient undergoing previous glaucoma surgery. Am J Ophthalmol. 1979 Jun;87(6):766-8.

9 Palamar M, Thangappan A, Shields CL, Ehya H, Shields JA. Necrotic choroidal melanoma with scleritis and choroidal effusion. Cornea. 2009;28(3):354-6.

10 Iturralde JC, Bianciotto C, Lally SE, Krasnow M, Shields CL. Massive choroidal effusion and painful secondary glaucoma from underlying uveal melanoma. Graefes Arch Clin Exp Ophthalmol. 2012 Apr 1;250(4):627-30.

11 Kivela T, Eskelin S, Makitie T, Summanen P. Exudative retinal detachment from malignant uveal melanoma: predictors and prognostic significance. Invest Ophthalmol Vis Sci. 2001 Aug;42(9):2085-93.

12 Mearza AA, Harsum S, Hiscott P, Chopdar A. Choroidal neovascular membrane associated with malignant melanoma presenting as angle closure glaucoma. Acta Ophthalmol Scand. 2004 Oct;82(5):628-30.

13 Kaliki S, Shields CL, Shields JA. Uveal melanoma: estimating prognosis. Indian J Ophthalmol. 2015 Feb;63(2): 93-102. 